\title{
Hierarchical models of plasticity: dislocation nucleation and interaction
}

\author{
Rob Phillips $\dagger$, David Rodney $\dagger$, Vivek Shenoy $\dagger$, Ellad Tadmor $\ddagger$ and \\ Michael Ortiz§ \\ $\dagger$ Division of Engineering, Brown University, Providence, RI 02912, USA \\ ‡ Division of Engineering and Applied Science, Harvard University, Cambridge, MA 02138, USA \\ $\S$ Department of Aeronautics, California Institute of Technology, Pasadena, CA 91125, USA
}

Received 5 December 1998, accepted for publication 3 March 1999

\begin{abstract}
One avenue being pursued in the development of dislocation-based models of plasticity is the explicit simulation of the dynamics of dislocations, based on the recognition that such dislocations are the carriers of plasticity. The construction of models of dislocation dynamics requires insights into both the nucleation and interaction of dislocations, many of the details of which fall outside the domain of validity of the linear theory of elasticity. The aim of the present paper is to show how preliminary steps have been made to elucidate the mechanisms of dislocation nucleation and interaction, and to illustrate how such information can be imported into explicit models of dislocation dynamics. This effort reflects, in part, the research program of Gilles Canova, to whom the present volume is dedicated and to whom the authors dedicate this paper.
\end{abstract}

\section{Hierarchical modelling in plasticity}

Plasticity has attracted attention from a number of different quarters, ranging from engineers strictly interested in a proper accounting of the deformation history of a material to those studying the physics of exotic materials, such as the vortex lattice in high-temperature superconductors. The traditional discipline of single-crystal plasticity has been built around a series of postulated phenomenological constitutive assumptions in which laws for initial yield and subsequent hardening are chosen in accordance with experimental observations while maintaining a reasonable level of computational tractability [1]. Such models explicitly acknowledge the underlying existence of dislocations only through the geometry of crystalline slip in which it is assumed that slip can only occur on certain planes and in certain directions.

On the other hand, a longstanding ambition of the theory of dislocations has been the construction of a theory of plasticity built upon insights into the fundamental properties of dislocations [2]. Despite the difficulties of constructing convincing analytic models involving the motions of many dislocations, one class of recent computational model has attempted to simulate plastic deformation directly on the basis of dislocation dynamics [3-7]. Such calculations represent an intriguing first attempt at an explicit construction of models of plasticity from the ground up, in which the governing defects themselves give rise to the mechanical properties with recourse to far fewer phenomenological intermediaries.

The theses set forth in the present paper reflect an approach that was elucidated by Gilles Canova, to whom the present volume is dedicated. The argument is that despite the desire to explicitly simulate the plasticity of materials on the basis of dislocation theory alone, there are a number of features of dislocation physics that remain uncertain, including a variety of nonlinear 
processes such as dislocation nucleation and junction formation between dislocations. As a result, a particularly appealing objective is the use of atomic level modelling to introduce such dislocation physics directly into dislocation dynamics models. This paper examines the use of the quasicontinuum method $[8,9]$ to explicitly characterize such 'subgrid' processes as dislocation nucleation and interaction with the aim of exporting these insights directly to the dislocation dynamics arena.

The remainder of the paper is organized as follows. We begin with a discussion of current models of dislocation dynamics, with special attention devoted to the missing information in such models. Section 3 considers dislocation nucleation under conditions of nano-indentation, with an eye to extracting useful criteria for these processes directly on the basis of atomic-level calculations. Section 4 sketches our recent work to examine the interactions of dislocations, again with the similar objective of linking the insights from these calculations directly to models of dislocation dynamics. In the final section, we take stock of how far progress has been made in closing the hierarchy proposed here, and call attention to the current impediments to further progress.

\section{Dislocation dynamics—fundamentals and conundrums}

A longstanding ambition in the analysis of deformation has been the construction of models of single-crystal plasticity predicated directly on the nucleation, motion and interaction of dislocations. One way to test a particular theoretical paradigm is to create a computational incarnation of that paradigm and to explore how closely it reproduces experimental observations. In the present setting, this means bringing together everything that is known about the 'many-body problem' of the dynamics of interacting dislocations, and to see if the virtual metal bears sufficient resemblance under deformation to real metals. For example, one might question whether the initial yield occurs at stress levels consonant with those measured experimentally, whether the subsequent hardening with increasing strain is the same as that observed in experiments, or whether the temperature dependence of the flow stress exhibits the same features as seen in experiments.

To construct a viable computational model of dislocation dynamics, it is necessary to assemble a variety of different mechanisms and interactions, since hardening is the result of collective processes involving many dislocations. First, it is necessary to be able to characterize the response of a given segment to an applied stress. From the perspective of traditional dislocation theory, this coupling is characterized by the elastic Peach-Koehler force,

$$
\boldsymbol{f}_{\mathrm{tot}}(i)=\left[\boldsymbol{\sigma}_{\mathrm{ext}}+\sum_{j \neq i} \boldsymbol{\sigma}(j)\right] \boldsymbol{b} \times \mathrm{d} \boldsymbol{l}
$$

where the stress acting on the segment of interest is a combination of the external stress $\sigma_{\text {ext }}$ and the stress on segment $i$ due to all the other segments $j, \sigma(j)$. This stress describes the collective effect of the rest of the dislocation segments on the segment of interest. The linearity assumption present in linear elasticity makes it possible to write this term in closed form as the sum of 'two-body' interactions. The deceptive feature of blithely writing down this expression is that once the distance between two segments is at the scale of the Burgers vector itself, the linear elastic model used to compute the interaction stress between two segments is no longer valid. As a consequence, various rules have been set forth that attempt to bypass this difficulty. Short-range dislocation interactions involve nonlinear effects that cannot be written in the simple linear elastic formalism described above. The solution is to use various phenomenological rules that force the nonlinear processes to occur. This requires an understanding of these effects prior to the simulations. In the case of junction formation, 
the rule used states that if two dislocations approach each other at a distance less than a certain minimum and elastically attract each other, they will either annihilate or form a junction, the strength of which is input into the model as an external parameter.

A similar quandary must be confronted in the context of dislocation nucleation. In this case, the dislocation dynamics models are again faced with the difficulty that there is no clear cut criterion which dictates the need to add another dislocation in the medium. In a recent dislocation dynamics simulation of nano-indentation [10], for example, new dislocation segments were added, as necessary, to ensure a fit between the simulated force-displacement curve and one measured experimentally for the same system. Again, it is clear that the predictive power of these simulations would be aided considerably by the introduction of mechanism-based criteria for nucleation.

Although this paper will examine the physics of two elemental processes involving dislocations, namely their nucleation and interaction, there are a number of other elemental processes which are important to dislocation dynamics, such as the annihilation of dislocation dipoles or their cross-slip [11,12], which may also be considered from a microscopic perspective. The various nonlinear mechanisms described above involve elemental processes in which only one or a small number of dislocations participate at a given time. Different methods can be used to quantitatively characterize these processes, including analytical calculations and the use of atomistic simulation techniques such as the quasicontinuum method. These ideas will be fleshed out in the following sections.

\section{Nucleation criteria for dislocation-based models of plasticity}

Much recent excitement in the mechanics of materials has attached to questions surrounding the conditions under which dislocations are nucleated. Nucleation events may occur in the induced plastic zone beneath an indenter, as a result of the loading of a crack tip, or in the context of heteroepitaxial films in which dislocations play a key role in strain relaxation.

From the standpoint of dislocation dynamics models of plasticity, the nucleation of dislocations remains something of a black art in the sense that injection of further dislocation loops into a given simulation is predicated on satisfying certain ad hoc criteria. The aim of the discussion in the present section is to illustrate the way in which both analytic arguments and the quasicontinuum method can be used to better characterize nucleation events in problems, such as that of nano-indentation. In particular, we focus on idealized geometries in face-centred cubic (fcc) crystals in which the indentation problem is essentially two-dimensional with the indenter extending indefinitely along one direction.

\subsection{Analytical models of indentation}

The geometry of the problem is illustrated in figure 1, where indentations are made on the

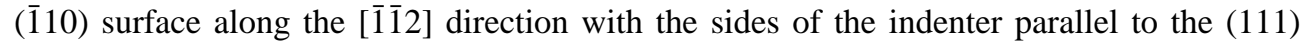
slip planes. For the purposes of the simplest argument that we will set forth, the indenter is idealized as a uniform pressure, $p_{0}$, in the region $-a \leqslant x \leqslant a$, where the $x, y$ and $z$

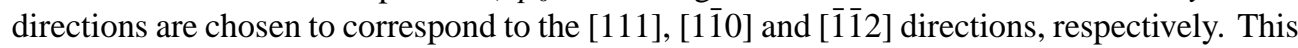
geometry is conducive to the nucleation of dislocations at $x= \pm a$, where adjacent (111) planes suffer a relative displacement along the [110] direction. While such an indenter is an analytical construction, the load distribution it produces lies in between that of the cylindrical hertzian indenter, which has a load distribution that is maximum at the centre of the region of contact and decreases towards the edges, and a rigid indenter which gives rise to a load distribution that has a minimum at the centre and diverges as the edges are approached [13]. 


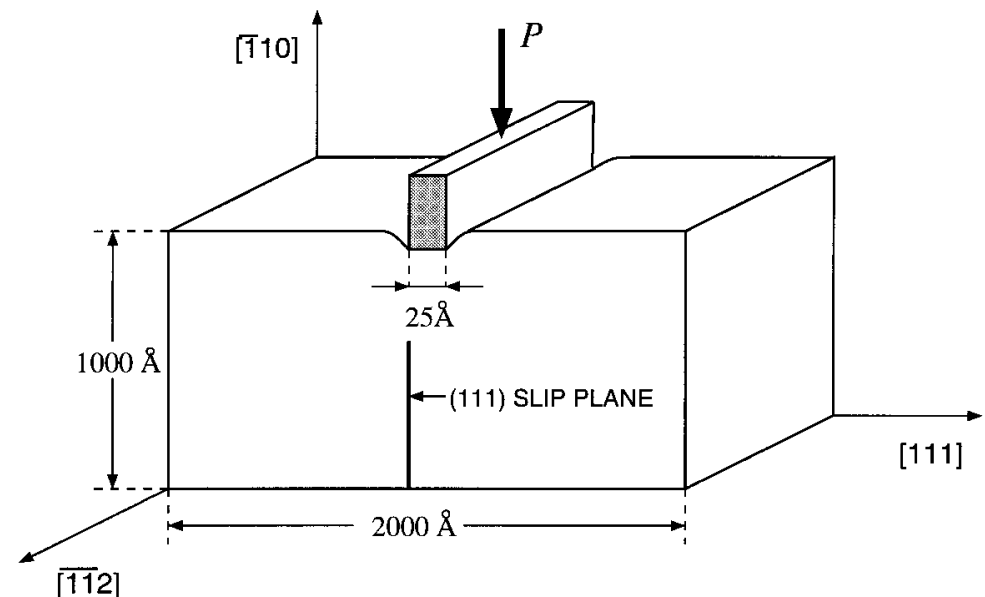

Figure 1. A schematic of the indenter geometry considered in our models of dislocation nucleation.

Furthermore, the choice of the load distribution and slip geometry enables us to derive closedform expressions for the stresses arising from both the slip distribution and the indenter load. As will be described elsewhere, a more faithful job can be done in treating the force distribution between the indenter, but only by recourse to numerical methods. In this section we examine the criterion for dislocation nucleation for the indentation problem using two standard approaches used to determine dislocation nucleation from crack tips [14, 15].

3.1.1. Rice-Thomson model for dislocation nucleation. We adapt the criterion derived by Rice and Thomson [14] for dislocation nucleation from a crack tip to the indentation problem. To do so, we introduce Volterra dislocations on the slip planes at $x= \pm a$ at a distance $d$ beneath the surface, and consider the $z$-component of the force acting on them. The force acting on the dislocation at $x=a$ can be written as $F_{z}=-b \sigma_{x z}(a, d)$, where $b$ is the magnitude of the Burgers vector and $\sigma_{x z}$ is the total shear stress acting on the dislocation. For the simplest case in which a uniform pressure $p_{0}$ is considered, the $x z$ component of the stress tensor has a contribution, $\sigma_{x z}^{\mathrm{L}}(a, d)=-\left(p_{0} / \pi\right)\left(4 a^{2}+z^{2}\right)$, arising from the indenter load which has a tendency to push the dislocation away from the surface. This contribution of the external loading to the total stress can be evaluated by using the Green function for a point load acting on the surface and summing up the various infinitesimal contributions arising from the region $\{-a, a\}$. The other contributions to the total shear stress are due to the presence of the dislocations at $x=-a$ and the images of both dislocations necessary to satisfy the free surface boundary conditions. These contributions tend to pull the dislocation towards the surface.

The competition between the external loading and the image stresses imply that if the dislocation is too close to the surface, it will be attracted to it, while if the dislocation is at a sufficient depth, it is injected into the solid. The Rice-Thomson (RT) strategy is to compute the position of unstable equilibrium (which depends upon the external load $p_{0}$ ) and to assume that nucleation occurs when this distance is equal to the dislocation core radius. The location $d_{0}$ where the dislocation is in unstable equilibrium is given by the solution to the equation

$$
\left(\frac{p_{0}}{\pi}\right) \frac{4 a^{2}}{d_{0}^{2}+4 a^{2}}-\left(\frac{\mu}{4 \pi(1-v)}\right) \frac{a^{6}+3 d_{0}^{2} a^{4}+6 d_{0}^{4} a^{2}}{\left(d_{0}^{2}+a^{2}\right)^{3}}=0 .
$$

By letting $d_{0}=r_{\mathrm{c}}$ we find the critical load, $P_{\mathrm{cr}}$, to nucleate a dislocation that would propagate 
into the bulk to be

$$
P_{\mathrm{cr}}=\frac{\mu}{4(1-v)}\left(\frac{b}{r_{\mathrm{c}}}\right)\left(1+\frac{r_{\mathrm{c}}^{2}}{4 a^{2}}\right)\left(\frac{a^{6}+3 r_{\mathrm{c}}^{2} a^{4}+6 r_{\mathrm{c}}^{4} a^{2}}{\left(r_{\mathrm{c}}^{2}+a^{2}\right)^{3}}\right) .
$$

In the limit when the indenter radius $a$ is much larger than the core radius $r_{\mathrm{c}}$, equation (3) reduces to $P_{\mathrm{cr}}=\mu / 4(1-v)$, where we have taken $r_{\mathrm{c}}=b$. In the following sections we will comment on this critical load, in light of both more sophisticated analytic models and numerical results using the quasicontinuum method.

3.1.2. Dislocation nucleation based on the Peierls concept. We now turn to a more sophisticated model for dislocation nucleation developed by Rice [15]. In this approach, instead of a fully formed dislocation, a slip distribution is allowed to occur along slip planes that are chosen based on the crystallography of the problem. At a certain critical load, the incipient slip distribution becomes unstable and leads to a fully formed dislocation. For the indentation problem under consideration, we assume that slip occurs along the planes $x= \pm a$, as shown in figure 2. A periodic relation between shear stress and atomic shear displacement, derived using the Ercolessi and Adams potential [16], is used in the calculations [17]. The slip distribution is expressed as

$$
\delta(z)=u_{z}\left(a_{-}, z\right)-u_{z}\left(a_{+}, z\right)=u_{z}\left(-a_{+}, z\right)-u_{z}\left(-a_{-}, z\right)
$$

where $u_{z}$ is the $z$-component of displacement.

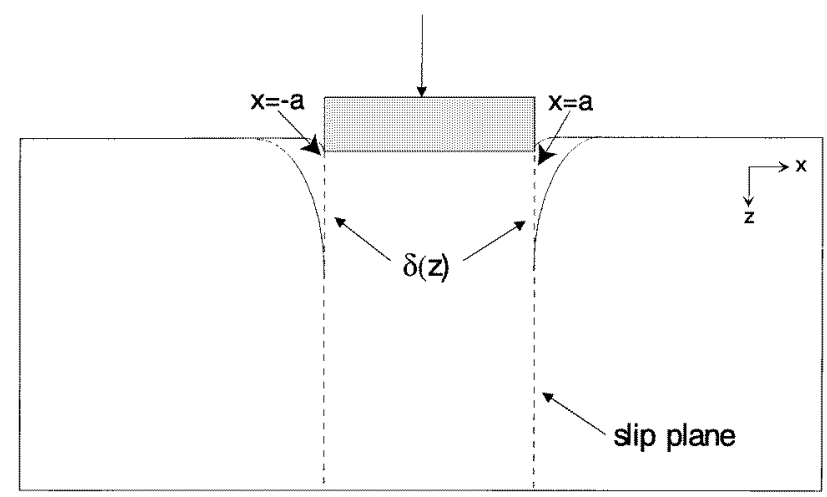

Figure 2. Illustration of the incipient slip distribution that arises beneath the indenter in response to loading.

To construct the energy of the slipped solid, let $U_{0}$ be the elastic energy of the loaded solid obtained from the linear elastic solution where $\delta$ is constrained to be zero. Following the reasoning considered by Rice in the crack-tip context [15], the slipped-configuration energy is given by $U[\delta(z)]=U_{0}+$ the energy of the slip planes due to the slip $\delta+$ the energy change of the linear elastic solid due to the introduction of the slip distribution. It can be shown that

$U[\delta(z)]=U_{0}+2 \int_{0}^{\infty} \Phi[\delta(z)] \mathrm{d} z+2 \int_{0}^{\infty} \sigma_{x z}^{\mathrm{L}}(a, z) \delta(z) \mathrm{d} z+\int_{0}^{\infty} \sigma_{x z}^{\mathrm{d}}(a, z) \delta(z) \mathrm{d} z$

where $\Phi[\delta(z)]$ is the interplanar potential evaluated at point $z$ where the adjacent planes have slipped by $\delta(z), \sigma_{x z}^{\mathrm{L}}$ is the stress field produced by the indenter and $\sigma_{x z}^{\mathrm{d}}$ reflects the stress fields produced by the slip distribution. The basic idea is to sum up all of the interaction energies 
between the participating infinitesimal dislocations. In terms of the stress fields produced at $(x, z)$ by a Volterra dislocation with unit Burgers vector located at $\left(x^{\prime}, z^{\prime}\right)$,

$\sigma_{x z}^{\mathrm{s}}\left(x^{\prime}, z^{\prime} ; x, z\right)=\frac{\mu}{2 \pi(1-v)}\left[\frac{z-\left[z_{-}^{2}-x_{-}^{2}\right]}{\left[z_{-}^{2}+x_{-}^{2}\right]^{2}}-\frac{z+\left[z_{+}^{2}-x_{-}^{2}\right]}{\left[z_{+}^{2}+x_{-}^{2}\right]^{2}}-\frac{2 z^{\prime}\left[z_{-} z_{+}^{3}-6 z z_{+} x_{-}^{2}+x_{-}^{4}\right]}{\left[z_{+}^{2}+x_{-}^{2}\right]^{3}}\right]$

where $z_{ \pm}=z \pm z^{\prime}$ and $x_{-}=x-x^{\prime}$, we can write

$$
\sigma_{x z}^{\mathrm{d}}(a, z)=\int_{0}^{\infty} \frac{\mathrm{d} \delta\left(z^{\prime}\right)}{\mathrm{d} z^{\prime}}\left[\sigma_{x z}^{\mathrm{s}}\left(-a, z^{\prime} ; a, z\right)-\sigma_{x z}^{\mathrm{s}}\left(a, z^{\prime} ; a, z\right)\right] \mathrm{d} z^{\prime} .
$$

Minimizing $U[\delta(z)]$, one finds that the slip distribution can be obtained by solving the integrodifferential equation.

$$
-\frac{\mathrm{d} \Phi}{\mathrm{d} \delta(z)}=\sigma_{x z}^{\mathrm{L}}(a, z)+\sigma_{x z}^{\mathrm{d}}(a, z)
$$

The equation considered above is for the as yet unknown slip distribution. An important feature of this equation is that for virtually no effort one can determine the slip at the indented surface, and this information alone suffices to reveal the nucleation criterion. An explicit expression for the value of slip at the edge of the indenter is obtained by noting that $\tau(\delta(0)) \equiv \partial \phi(\delta(0)) / \partial \delta=p_{0} / \pi$, since $\sigma_{x z}^{\mathrm{d}}(a, 0)=0$. This implies that incipient slip distribution becomes unstable leading to the formation of a full dislocation when the load reaches the critical value $P_{\mathrm{cr}}=\pi \tau_{\max }$. Here, $\tau_{\max }$ is the maximum shear stress that occurs when two planes are rigidly displaced with respect to each other and can be extracted simply from atomistic calculations of the relevant $\gamma$-surface. On the assumption that the interplanar potential is specified by the Frenkel sinusoid, $\tau_{\max }=\mu / 2 \pi$. Note that just before the nucleation of a fully formed dislocation, $\delta(0) \approx b / 4$ in contrast with the value of $b / 2$ that occurs in the nucleation criterion for a dislocation emerging from the tip of a crack. Also, the unstable stacking energy $\gamma_{u s}$, which is a key parameter in determining the load for nucleating dislocations at the crack tip, does not play a role in the indentation problem. Instead, the material parameter $\tau_{\max }$ determines the critical load for nucleating dislocations.

Both the RT and Peierls-Nabarro approaches have yielded interesting insights into the critical load for dislocation nucleation in the simplified two-dimensional geometry considered here. The Peierls-Nabarro estimate for the nucleation stress is highly provocative in that it suggests a simple scheme for introducing a nucleation criterion into the dislocation dynamics setting. One of the objectives of the next section is the consideration of numerical simulations of the same processes, with the aim of examining the validity of the analytic solutions developed above.

\subsection{Quasicontinuum simulations of dislocation nucleation}

Our preliminary efforts with nano-indentation have all been carried out in a pseudo-twodimensional framework in which the indenter is modelled as an infinite two-dimensional rigid bar. Although the geometry is intrinsically two-dimensional, as will be shown below, in some cases the dislocations that are nucleated beneath the indenter involve out-of-plane displacements. In this paper, our aim is to illustrate the way in which the quasicontinuum method may be used to determine the critical load at which nucleation occurs and the geometric character of the dislocations that are produced, while the details of these calculations will appear elsewhere [18]. Note that these numerical calculations consider similar indenter geometries and nucleation conditions to those that were considered in the analytic calculations presented above. Our ambition in the present setting is to make contact with the hierarchical modelling of dislocation-induced plasticity described earlier. 


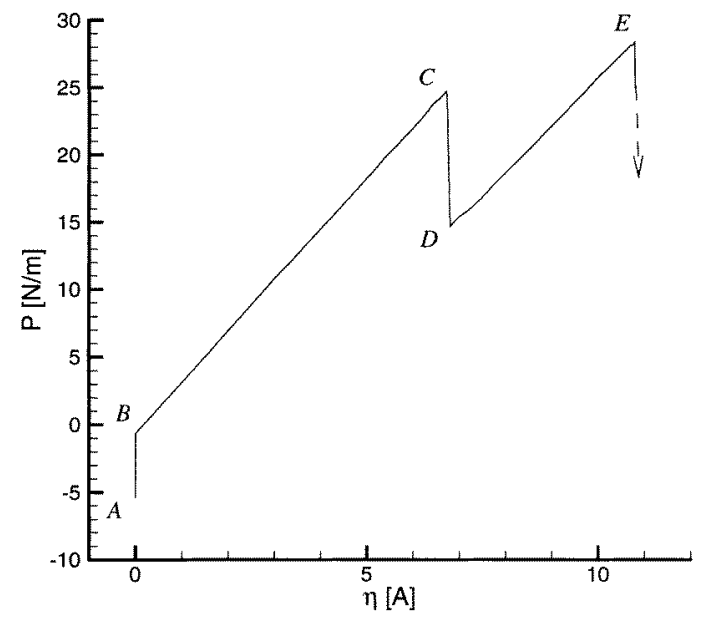

Figure 3. The force-displacement curve associated with pseudo-two-dimensional nanoindentation in $\mathrm{Al}$.

Unlike the analytic solutions presented above, in the quasicontinuum simulations a displacement boundary condition is applied to those surface nodes that are assumed to be in contact with the indenter. The calculations are carried out as a series of static energy minimizations in which at each step a particular displacement of the indenter is prescribed, and the energy is then minimized. The force-displacement curve can be monitored as the process continues, as can the atomic positions beneath the indenter, with the result that the critical event, namely dislocation nucleation, can be easily identified.

The force-displacement curve associated with the sequence of calculations of interest here is shown in figure 3. In addition, the atomic-level geometry beneath the indenter after dislocation nucleation is shown in figure 4. From the point of view of passing nucleation information to higher-level dislocation dynamics codes, these results are useful as follows. If we take as our paradigm the two-dimensional calculations of Cleveringa et al [19], the dislocation dynamics treatment of nano-indentation would amount to the solution of a boundary value problem. However, as the indenter is pressed to increasing depths, such models are faced with a critical uncertainty. At what stage in the model should new dislocations be introduced to accommodate the deformations induced by the indenter?

Both our analytic and numerical calculations provide valuable clues into how a nucleation criterion might be incorporated into higher level dislocation dynamics codes. The analytic solutions given above suggest that when the shear stress at the surface on the relevant slip plane reaches a critical value, new dislocations are nucleated. To make this connection between the analytic and numerical calculations more concrete, the uniform pressure assumed in the analytic calculations described here must be replaced with a spatially varying distribution that can be extracted from the quasicontinuum simulations. Our present work is devoted to shoring up the connection between the analytic and numerical models of nucleation.

\section{Interaction strengths for dislocation-based models of plasticity}

At large distances, the interactions between dislocations are captured satisfactorily on the basis of the linear theory of elasticity. However, at short distances, the interactions can no longer be described on the basis of conventional elasticity theory, and this necessitates the formulation 

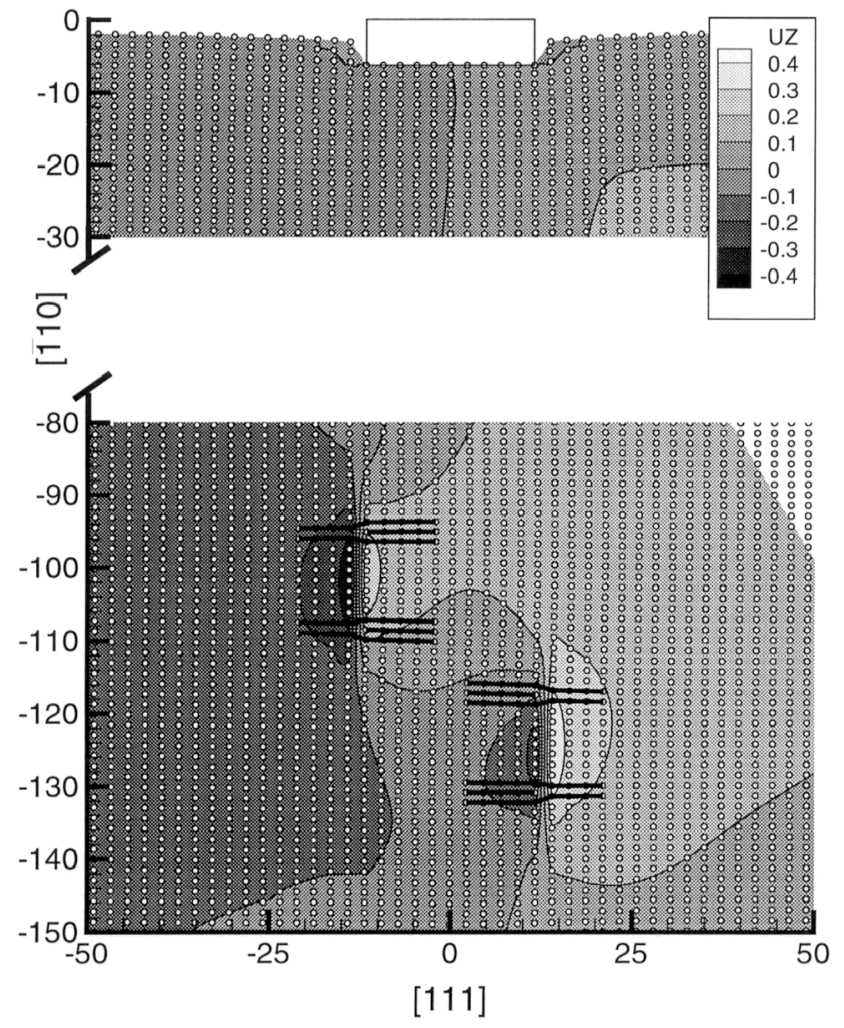

Figure 4. The region just beneath the indenter illustrating the presence of a series of indentation induced partial dislocations. Contours indicate out-of-plane displacement in $\AA$.

of rules for handling such interactions. In particular, one is forced either to posit that the elastic interactions are the only interactions that occur, or to resort to simple rules such as the statement that if two dislocations are closer than some minimum distance, they will form a junction that is characterized by a strength that is put in by hand.

On the basis of atomic-level calculations, it is conceivable that the strength of the type of short-range interactions discussed above could be parametrized directly. This forms the basis of our current efforts to fill the gap in the hierarchy of schemes used to model plasticity. The essential idea is to compute the structure and energetics of junctions, both in the absence of and presence of an applied stress. Here we focus on fcc aluminum as described by the Ercolessi and Adams potentials. Junctions are dislocation segments common to two or more dislocations. Symmetry arguments alone suffice to demonstrate that there are only six possible junctions that can form between perfect $a / 2\langle 110\rangle\{111\}$ fcc distribution in these materials. For the purposes of the present discussion, we will concentrate on the Lomer-Cottrell lock, which is the junction thought to be the predominant factor in hardening of fcc materials [20].

We note again that, in the context of dislocation dynamics models, the strengths of junctions like that of the Lomer-Cottrell lock are external inputs. Our intention is to begin to establish an atomistic basis for characterizing the strength of such junctions. The advent of increasing computational power has recently begun to make such calculations possible [21-23]. To calculate the structure of the Lomer-Cottrell lock in the absence of an applied stress, we begin with an initial configuration comprising two Volterra dislocations pinned at 


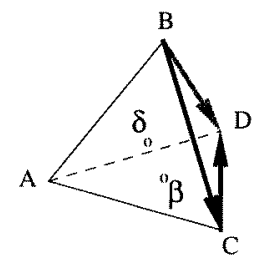

(a)

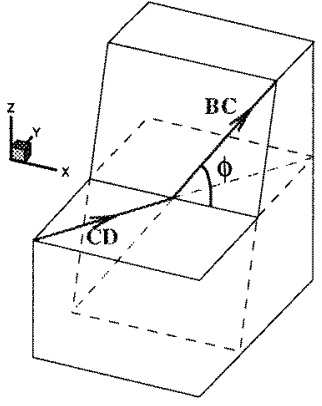

(b)

Figure 5. (a) The Thompson tetrahedron illustrating the Burgers vectors of the dislocations of interest. (b) A schematic section of the initial simulation cell along the glide planes of the dislocations. The two dislocations have the same length, $2 l$, and make the same angle $\phi$ with the line of intersection between the glide planes.

the surfaces of the computational cell as shown in figure 5(b). The Volterra dislocations used to construct the boundary conditions are perfect fcc dislocations which reside on different $\{111\}$ glide planes, intersecting along the $x$-axis of the simulation cell. The Burgers vectors of the dislocations are represented in the Thompson tetrahedron of figure 5(a). Our choice of simulation geometry was motivated largely by the desire to make direct contact with the elastic models of junction formation such as those proposed by Saada over 30 years ago [24, 25]. The highly symmetric configuration considered here is identical to the one considered in Saada's line tension model for junction formation; in it both the dislocations make the same angle with the line of intersection between the glide planes. Note that although the junction geometry is already characterized at the level of the participating slip systems by the slip plane normals and Burgers vectors, there are additional degrees of freedom associated with the junction which are not considered in the geometric classification. In particular, the orientation of the dislocation line itself can play a role in the nature of the resulting junctions.

To consider the action of an external stress on the junction, a series of strains are imposed upon the simulation box. These shear strains can be translated into corresponding stresses by assuming a linear elastic relation between stress and strain. The result of the increasing stresses is to bow out the junction segments, to introduce a net translation of the junction segment itself and, ultimately, to unzip it. A series of snapshots of the junction geometry are shown in figure 6 . The plots are constructed by showing only those atoms with an energy higher than a certain threshold. For the particular geometry shown here, the sequence of snapshots illustrates the unzipping mechanism whereby the junction is dissolved, allowing for one dislocation to pass through the other. From the standpoint of dislocation dynamics models, the junction strength itself is of particular interest. Unfortunately, there are still unresolved questions surrounding the quantitative determination of the strength which arise largely from the fact that it is unclear how to handle the boundary conditions without producing spurious forces as the junction unzips.

Figure 6(a) presents the structure in the absence of any applied stress. A junction forms along the line of intersection between the glide planes. This structure is consistent with tunnelling electron microscopy observations of Lomer-Cottrell junctions made in lowstacking-fault-energy $\mathrm{CuAl}$ alloys [26]. From a quantitative standpoint, we aim to estimate the stress required to destroy this junction and force the dislocation in the horizontal plane to cross 


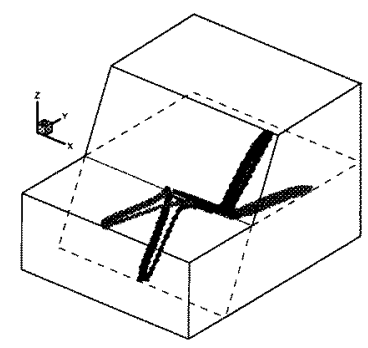

(a)

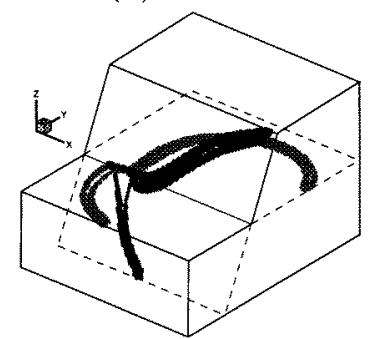

(c)

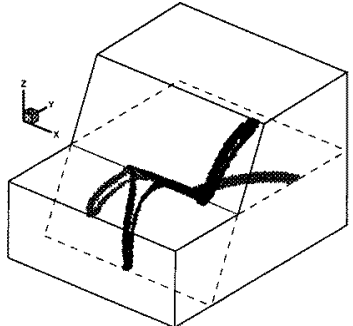

(b)

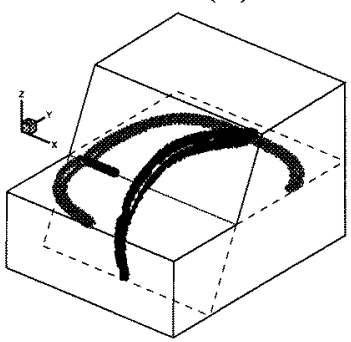

(d)

Figure 6. A sequence of snapshots of the junction geometry under increasing stress. (a) Zero applied stress, (b) the stress is $0.011 \mu$, (c) the stress is $0.018 \mu$ just before the junction breaks and (d) the stress is $0.018 \mu$ at the end of the simulation.

the other dislocation. The result of the increasing stresses as shown in figures 6(b)-(d) is to bow out the different arms that are connected to the junction segment itself and to gradually unzip the junction. Unzipping takes places as the two dislocations forming the junction dissociate under the action of the applied stress. This mechanism is precisely that supposed in the elastic models, although we should note that for a different choice of different initial line directions, an entirely different breaking mechanism has been obtained [27]. Once the junction is broken, the horizontal dislocation continues to bow out as a result of the applied stress, leaving behind a tube of energetic atoms that can be seen in figure 6(d). This tube is built of vacancies produced because of the non-conservative motion of the jog that forms on the horizontal dislocation when the junction breaks. The formation of this jog was expected on the basis of arguments from dislocation theory [28] and originates from the discontinuity in the displacement field associated with the Burgers vector of the other dislocation. The other dislocation also acquires a jog which is constricted and glissile. Its motion can be followed in the simulations. The simulation shows that the critical resolved shear stress for breaking the junction is $\sigma_{\mathrm{c}}=0.8 \mu \mathrm{b} / \mathrm{l}$ where $l$ is the initial length of the junction arms. This value is of the same order of magnitude as those determined on the basis of linear elasticity. This calculation is a preliminary attempt to quantitatively evaluate the critical forces associated with junction dissolution, but many unresolved questions remain, some of which will be described in the following section.

\section{Finishing the job}

One of the strategies that has evolved to face the challenge of constructing dislocation-based models of plasticity is that of following the trajectories of the individual dislocations that populate a given material. As noted above, for such a strategy to be effective, a means must 
be found to characterize those effects that fall outside of the purview of the elastic theory of dislocations, such as the nucleation and interaction of dislocations. Although the present paper has sketched the rudiments of how such information may be passed from atomiclevel simulations and analytical modelling to dislocation dynamics, a number of important challenges remain.

To close the hierarchy described here, essential progress is necessary on two main fronts. At the level of the atomic-scale simulations, it is essential to carry out full three-dimensional calculations for examining both nucleation and interaction, with well controlled boundary conditions. The nucleation calculations carried out here were all essentially two-dimensional. The next development will be to carry out three-dimensional nano-indentation calculations, with special emphasis on the geometry of the loops produced in such calculations, and the conditions under which such loops are nucleated [29]. From the standpoint of our efforts to understand dislocation interactions, the calculations presented here are found wanting on the grounds that the boundary conditions are essentially uncontrolled and appear to impact their quantitative outcome, necessitating further efforts along these lines. In particular, the dislocation junctions simulated here bow out in the presence of boundary conditions which exert unphysical forces on these segments. Our current efforts are aimed at removing the influence of these boundary effects. In addition to problems with boundary conditions, because we inherit a stacking fault energy from our interatomic potentials that is lower than that observed experimentally in $\mathrm{Al}$, the material specificity of our calculations is also spoiled.

The second missing link in the effort to pass mechanistic information to the dislocation dynamics methods concerns the more conceptual question of precisely what information one wishes to draw from the atomic-scale calculations. For example, in the context of nucleation, once a series of simulations on the atomic-scale mechanisms of nucleation have been carried out, what generic rules can be passed to the dislocation dynamics setting? Similarly, is the current practice of inserting rules for the formation and dissolution of junctions appropriate, or should one seek a means of passing smoothly between the linear elastic treatment of the dislocations and their nonlinear counterparts which signal the onset of junction formation? These questions and others like them will form a significant thread in coming research on hierarchical modelling of plasticity, efforts that will miss the insights of Gilles Canova.

\section{Acknowledgments}

The authors would like to express their sorrow at the untimely death of Gilles Canova and to dedicate this paper to the memory of many happy hours spent in his company, and to his family, Marie, Sandrine, Lauren, Antoine and Mathieu. In addition, we are grateful to Glen Beltz for many discussions and the use of his code, and to J Bassani, E van der Giessen, M Fivel and $\mathrm{C}$ Robertson for numerous discussions on implementing the type of hierarchy described here. We are also grateful for support from Electricité de France, the NSF through the Brown University MRSEC program and a Career Award, and the ASCI Center at Caltech.

\section{References}

[1] Bassani J L 1994 Adv. Appl. Mech. 30 191-258

[2] Rice J R 1970 J. Appl. Mech. 37728

Rice J R 1971 J. Mech. Phys. Solids 19433

[3] Fivel M, Verdier M and Canova G 1997 Mater. Sci. Eng. A 234-236 923

[4] van der Giessen E and Needleman A 1995 Modelling Simulation Mater. Sci. Eng. 3689

[5] Zbib H M, Rhee M and Hirth J P 1998 Int. J. Mech. Sci. 40113

[6] Devincre B, Veyssiere P, Kubin L P and Saada G 1997 Phil. Mag. A 751263 
[7] Devincre B and Kubin L P 1997 Mater. Sci. Eng. A 234-236 8

[8] Tadmor E B, Ortiz M and Phillips R 1996 Phil. Mag. A 731529

[9] Shenoy V B, Miller R, Tadmor E B, Phillips R and Ortiz M 1998 Phys. Rev. Lett. 80742

[10] Fivel M C, Robertson C F, Canova G R and Boulanger L 1998 Acta Mater. 466183

[11] Rasmussen T, Jacobsen K W, Leffers T and Pederson O B 1997 Phys. Rev. B 562977

[12] Duesbery M S 1998 Modelling Simulation Mater. Sci. Eng. 635

[13] Johnson K L 1985 Contact Mechanics (Cambridge: Cambridge University Press)

[14] Rice J and Thomson R 1974 Phil. Mag. 2973

[15] Rice J R 1992 J. Mech. Phys. Solids 40239

[16] Ercolessi F and Adams J 1994 Europhys. Lett. 26583

[17] Miller R and Phillips R 1996 Phil. Mag. A 73803

[18] Tadmor E B, Miller R, Phillips R and Ortiz M 1999 J. Mater. Res. in press

[19] Cleveringa H M M, van der Giessen E and Needleman A 1997 Acta Mater. 453163

[20] Franciosi P, Berveiller M and Zaoui A 1980 Acta Metall. 28273

[21] Bulatov V, Abraham F F, Kubin L, Devincre B and Yip S 1998 Nature 391669

[22] Zhou S J, Preston D L, Lomdahl P S and Beazley D M 1998 Science 2791525

[23] Baskes M I, Hoagland R G and Tsuji T 1998 Modelling Simulation Mater. Sci. Eng. 69

[24] Saada G 1960 Acta Metall. 8841

[25] Schoeck G and Frydman R 1972 Phys. Status Solidi 53661

[26] Karnthaler H P and Wintner E 1975 Acta Metall. 231501

[27] Rodney D and Phillips R 1998 Phys. Rev. Lett. 821704

[28] Heidenreich R D and Schockley W 1948 Bristol Conference, Phys. Soc. (London)

[29] Preliminary efforts in this direction have been undertaken by Kelchner C and Hamilton J 1998 Phys. Rev. B 58 11085 